\title{
Enseignements Dispenses Dans Le Systeme Educatif Ivoirien Et Les Theories D’apprentissage: Quelle Pertinence?
}

\author{
Djelle Opely Patrice-Aime \\ Ecole Normale Supérieure d'Abidjan \\ (REPUBLIQUE DE COTE D’IVOIRE)
}

doi: 10.19044/esj.2016.v12n7p479 URL:http://dx.doi.org/10.19044/esj.2016.v12n7p479

\begin{abstract}
This article is aimed at discussing the relevance of the education system face to learning theories. It will check in what ways our approaches are both relevant and logical. Then show that three steps methodology used here must at the same time combine quantiative accuracy, group survey and the interview. And also show that the results are congruent with the problematic. In general the Ivorian Education System partly meet operalization and complex cognitive proceedings developed by individuals. A lot has to be done to bring about change, for this to happen pedagogical renewal taking into account current learning theories is needed
\end{abstract}

Keywords : learning,pedagogical,interacivity,cognition and relevance.

\section{Resume}

Nous discuterons dans cet article de la pertinence du système éducatif face aux théories de l'apprentissage. Nous essaierons de voir en quoi nos démarches sont pertinentes, sont logiques. La méthodologie en trois étapes doit allier à la fois les précisions quantitatives, l'enquête de groupe et l'entretien. Les résultats obtenus correspondent à la problématique posée. Le système éducatif ivoirien en général répond partiellement à l'opérationnalisation et l'analyse des processus cognitifs complexes qui sont développés par les sujets. Beaucoup reste à faire. Pour cela, il faut s'attendre à un changement c'est-à-dire, à un renouveau pédagogique qui tienne compte des théories en cours.

Mots clés : Apprentissage, pédagogique, interactivité, cognition et pertinence. 


\section{1-Introduction}

Dans le processus d'appropriation de la culture, la maîtrise des systèmes symboliques est primordiale. Vygotski va s’intéresser à l'utilisation d’instruments en lumière avec la parenté entre certains comportements animaux et les comportements humains. Il adjoint la thèse d'outils spécifiques qu'il nomme symboles à celle d'une thèse classique appelé outils spécifiques (Brossard, 2004). Pour Vygotski, alors que l'instrument technique est tourné vers l'objet (le terme de symbole remplace celui d'instruments psychologiques), l'instrument psychologique est tourné vers soi et exerce son influence sur le psychisme propre. C'est ce «prêt de conscience» (Bruner, 2000) qui accompagne l'enfant au travers de la Zone de proche Développement. Dans le cadre théorique de Vygotski, c'est à un véritable tandem auquel l'on assiste entre l'expert et le novice par le biais de l'interaction sociale.

L'acte d'enseigner y est donc central. C'est l'enseignant qui dit et montre le savoir, le construit et le structure. Il n'y a rien à apprendre lorsqu'il ne parle pas ou ne montre pas. L’élève, lui, écoute attentivement et reçoit le savoir dans sa tête supposée vide. Il est modelé de l'extérieur et doit s'adapter aux activités magistrales ou interrogatives proposées par l'enseignant dans une situation de communication collective et verticale. En conséquence, un enseignement parfaitement réussi serait un exposé où l'enseignant ne commettrait aucune erreur, suivi d'un test où l'élève montrerait par des réponses justes qu'il a parfaitement compris. C'est le modèle “j'apprends/ j'applique”.

Pour lui, l'enfant réélabore le concept qu'il assimile, lui imprimant au cours de sa réélaboration les particularités de sa propre pensée, car poursuit Vygotski (1997) "la thèse selon laquelle l'enfant acquiert dans le processus d'apprentissage scolaire, les concepts tout prêts et les assimile comme on assimile n'importe quelle habileté intellectuelle est totalement dénuée de fondement".

Vygotski développe une théorie originale des rapports entre développement et apprentissage et selon cette perspective les apprentissages anticipent le développement. Il est à noter qu'il existe une indépendance complète entre le processus de développement et celui de l'apprentissage et partant d'une nette séparation des deux processus dans le temps (Schneuwly et Bronckart, 1985). Mais, l'apprentissage suit toujours le développement dans la mesure où certaines fonctions doivent arriver à maturation avant que l'école ne commence à enseigner à l'enfant des connaissances.

On se développe parce que l'on rencontre la contingence et la nécessité. C'est en faisant face aux situations imprévues et aux incidents qu'on modifie ses schèmes ou qu'on en développe de nouveaux: c'est l'apprentissage par adaptation (Clot, 2002). La contingence est une idée 
complémentaire de celle d'adaptation car l'une ne va pas sans l'autre. De plus, lorsque l'enfant apprend à lire et à écrire, ses fonctions psychiques (mémoire, attention, volonté...) sont encore immatures, seule sa capacité d’écrit suscitera, guidera le développement de ces fonctions vers de nouvelles formes. Favoriser les acquisitions chez l'enfant consiste pour l'adulte à aménager la transition de l'activité en tutelle à l'activité en autonomie et cela est fondé sur les interactions enseignant-enseigné.

C'est un paradoxe apparent sur lequel il nous faut insister: une autonomisation du développement succède à une interdépendance sociale, interdépendance qui fonde cette autonomisation (Vygotski, 1997). La possibilité plus ou moins grande qu'a l'enfant de passer de ce qu'il sait faire en collaboration avec quelqu'un à ce qu'il sait faire désormais tout seul, est précisément le témoignage en plus éclatant qui caractérise la dynamique de son développement et la réussite de son activité intellectuelle. Elle coïncide entièrement avec sa zone proximale de développement. La médiation sociale intervient dans le développement cognitif au cours des activités d'apprentissage, car l'éducation actualise les potentialités que l'enfant peut atteindre avec l'aide d'autrui et après par des exercices tout seul. La richesse et la plasticité des ressources cognitives mobilisables est un ingrédient essentiel de la compétence. Il convient de s’interroger sur ce que recouvre cette différente notion ainsi que les notions implicites.

Mais, quoi qu'il en soit, ses choix seront autant de réponses partielles et temporaires à la question cruciale de la psychologie de l'éducation : « comment apprend-on ? »; ou à celle, voisine mais non semblable, de tout praticien de l'enseignement : " comment favoriser l'apprentissage du plus grand nombre, sans faire obstacle aux apprentissages à venir, dans le temps imparti ? ».

Piaget (1967) souligne comme un résultat important de ses travaux la faillite expérimentale de cette conception transmissive qui confond apprentissage et enseignement. En effet, ce modèle sous-estime le rôle de l'élève et de ses processus cognitifs dans la construction de son savoir. Il ne laisse aucune autonomie à l'élève en dehors des phases de réinvestissement. Il prétend que le sens du message que l'enseignant pense communiquer est le même que celui que l'élève croit percevoir. De nombreuses études montrent qu'il n'en est rien. Elles montrent aussi que l'esprit n'est pas assimilable à une cire vierge.

Rejetant toute référence à la conscience, le behaviorisme s'applique à étudier scientifiquement le comportement de l'animal ou de l'homme défini comme « l'ensemble des réactions objectivement observables qu'un organisme généralement pourvu d'un système nerveux oppose aux stimuli, eux aussi observables, dans le milieu dans lequel il vit ». Il est envisagé comme un processus mécanique dans lequel les comportements de 
l’apprenant sont déterminés par les renforcements rencontrés : les "bonnes" réponses sont récompensées et reproduites, les "mauvaises” réponses punies et abandonnées. C’est l'apprentissage par conditionnement. Dans celle-ci, l'individu est actif, apprend en observant les conséquences de ses actes et en recevant des renforcements. Si le comportement procure du plaisir, il sera reproduit. Sinon, il sera abandonné. Cette conception de l'apprentissage a donné à Skinner les principes de l'enseignement programmé. Celui-ci consiste pour l'enseignant à proposer des situations où la matière à enseigner est découpée en unités aussi élémentaires que possible.

Ces situations doivent permettre à l'élève d'agir, de le faire travailler par étapes, et de renforcer au fur et à mesure ses acquisitions dans le sens d'une modification des comportements programmés par l'enseignant. Nous dirons comme Montmollin (1986), que les compétences attendues, seraient des ensembles stabilisés de savoirs et savoir-faire, de conduitestypes, de procédures standards et de types de raisonnement que l'on peut mettre en œuvre sans apprentissage. La compétence est toujours construite, c'est pourquoi Jonnaert et Vander Borght (2003) pensent, bien à propos, que la compétence serait comme l'intelligence des situations. De l'analyse de la comparaison de différentes définitions du concept de compétence, il ressort au moins trois éléments constants (Jonnaert, 2002):

- Une compétence reposerait sur la mobilisation et la coordination, par une personne en situation, d'une diversité de ressources,

- Une compétence ne se développerait qu'en situation,

- Une compétence ne serait atteinte que dans le cas d'un traitement achevé de la situation.

Est-ce que par rapport aux enseignements dispensés cela amène les apprenants à être compétents ? Les apprenants sont-ils devenus pertinents ? Sont-ils logiques par rapport à ce que l'on attendait d'eux ? Quel type d'évaluation peut-on en faire des enseignements dispensés en rapport aux théories d'apprentissage?

Comme hypothèse nous avançons que les apprenants à partir des enseignements dispensés dans le système éducatif ivoirien ceux-ci sont caractérisés par leur pertinence.

\section{2-Méthodologie}

Le contenu méthodologique pour l'atteinte d'un tel objectif est très important. Etant donné l'envergure de cette recherche, une méthodologie rigoureuse et adaptée est plus que nécessaire. Pour l'obtention des informations souhaitées, une méthodologie en trois étapes serait plus adaptée. Ces trois étapes doivent allier à la fois la précision quantitative et la qualité qualitative. Ainsi, nous pourrons aboutir à des résultats plus robustes 
en respectant rigoureusement une telle méthodologie, dont voici les déclinaisons :

\section{2-1-Des entretiens de groupe ou "FOCUS GROUPS "}

Ces entretiens ont pour but de rassembler des groupes homogènes (des enseignants ou des élèves) afin de qualifier leurs appréhensions du concept d' « enseignement » et celui d' « apprentissage ». Quatre entretiens avec d'une part des enseignants (deux groupes de professeurs enseignants différentes matières) et de l'autre des élève (deux groupes assez homogènes en terme de niveau d'étude). Pour des exigences de la méthode, chaque entretien se fera avec au plus 8 participants en plus du modérateur. La première partie qui concerne les enseignants, fournira une description de leurs méthodes d'enseignements, des innovations qu'ils introduisent de même que le fondement théorique de leurs méthodes. Avec les élèves, la discussion consistera en une description des méthodes d'enseignement en classe dans différentes matières ainsi que des méthodes de rétention. Comment ceux-ci se projettent où arrivent à se fixer des repère dans les cours qui leurs sont dispensés ? Comment ils construisent leur apprentissage et quelle perception ont-ils de l'enseignement et de l'apprentissage ?

\section{2-2-Une enquête quantitative}

Après la qualification des différentes variables qui nous intéressent à savoir la conception de l'enseignement aussi bien chez les élèves que chez les enseignants eux-mêmes, la conception de l'apprentissage, le lien que les enseignants ont avec les théories d'apprentissage, etc., cette permettra de quantifier l'information afin d'en avoir une mesure exacte pour l'établissement Jean-Piaget.

\section{2-3-Des mises en situation}

Cette ultime étape ou étape de contrôle permettra de vérifier d'une part les informations collectées dans les deux premières étapes et d'autre part au chercher d'apporter une caution critique à la vraisemblance de l'attitude de chaque partie eu égard à la théorie. Elle consistera à faire une ou plusieurs mises en situation. Le chercheur en assistant à différents cours, en prenant des notes sans intimider l'une ou l'autre des deux parties permettra de corriger voire améliorer la mesure faite plus haut par des éléments qualitatifs. La perception de certaines thèses d'apprentissage et leur application parmi les méthodes de l'enseignant (Thèse constructiviste, thèse behavioriste, thèse de Vygotsky et la thèse du traitement d'information)

- Les mesures pour une convergence certaine vers cet apprenant dans le système éducatif ivoirien 
- Les mesures pour une parfaite adéquation entre l'enseignement et l'apprentissage; en d'autres termes comment amener les apprenants à être compétents selon les enseignants.

- Les mesures pour une convergence certaine vers cet apprenant dans le système éducatif ivoirien.

Ces questionnaires ont été soumis à de faibles échantillons tant chez les enseignants que chez les élèves. Pour cette étude, 15 enseignants et 26 élèves ont répondu à nos questions (Le détails de ces questions est donné en annexe). Ce rapport est donc une synthèse des informations recueillies à cet effet. Dans un premier temps nous adopterons une approche centrée sur l'enseignant, puis nous nous intéresserons à l'apprenant. Avant d'aborder les différents points de vue des enseignants, il convient de décrire leur population. La population est définie comme l'ensemble des individus ayant en commun une caractéristique particulière.

\section{3-Resultats}

Les répondants aux questionnaires; pour ce qui concerne l'enseignement; enseignent dans 9 matières différentes. La collecte d'information a vu la participation de deux enseignants pour l'Anglais, les SVT, les Sciences-physiques, la Philosophie, les Mathématiques et d'Histoire-Géographie. Pour l'EPS, l'Espagnol et le Français, on enregistre la participation d'un seul enseignant. Pour ce qui est du genre des répondants, deux tiers des répondants sont des hommes tandis qu'un tiers est constitué de femmes.

Ensuite les enseignants devaient juger, sur la base de leur expérience, la validité d'une approche ou d'une théorie. Ils devaient ensuite donner leur compréhension de cette théorie et faire un rapprochement à leurs méthodes utilisées en mentionnant s'ils appliquaient la théorie en question.

Quatre théories d'apprentissage ont été retenues. Il s’agit de la Thèse constructiviste, la Thèse behavioriste, la Thèse de Vygotsky et la Thèse du traitement de l’information. Les résultats sont présentés par théorie.

\section{3-1-Rapprochement aux théories constructivistes de l'apprentissage $>$ Thèse constructiviste}

Selon cette thèse, "On apprend en agissant sur les choses et les gens qui nous entourent. Et selon ce qui se passe suite à notre action, on en tire des lois sur la structure de notre environnement. On apprend par l'action »

L'avis sur la validité de cette thèse et donné sur le graphique suivant : 


\section{Thèse constructivistes}

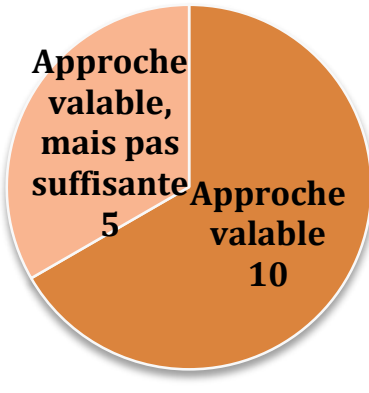

Pour deux tiers des enseignants, cette approche est entièrement valable. Cependant, le tiers des enseignants estime qu'elle n'est pas suffisante pour décrie le processus d'apprentissage. Dans les mêmes proportions, les enseignants affirment pratiquer cette théorie dans leurs méthodes.

\section{Application : Thèse constructivistes}

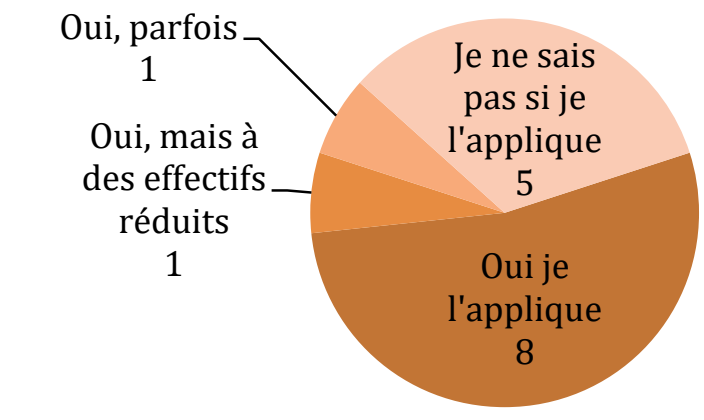

L'application de cette théorie n'est pas systématique pour deux enseignants. Le premier affirme l'utiliser souvent tandis que le second ne l'applique que pour des effectifs réduits.

Par ailleurs, le tiers des enseignants n'arrive pas à établir une correspondance claire entre leur manière d'emmener l'apprenant à un apprentissage pertinent et cette théorie. Deux facteurs peuvent expliquer ce constat. Le premier est que l'enseignant ne se soit pas clairement prononcé quant à l'application d'une telle théorie. Le second facteur réside essentiellement dans la compréhension de cette thèse. 


\section{3-2-Rapprochement aux théories behavioristes de l'apprentissage \\ Thèse behavioriste,}

Cette thèse avance que: "On met surtout l'emphase sur les conséquences du comportement. Ce qui compte pour Skinner, ce sont les 'contingences de renforcement', c'est-à-dire le type d'interaction entre l'individu et son environnement et le bénéfice que l'individu peut tirer. Si une action donnée a des conséquences bénéfiques, elle va s'installer de façon durable. Si ces conséquences sont néfastes, elle disparaîtra dans le répertoire de comportements de l'individu. Ce n'est pas l'apprenant qui construit son savoir de façon autonome. C'est le formateur qui programme ».La validité de cette thèse est illustrée sur le graphique suivant :

\section{Thèse behavioriste}

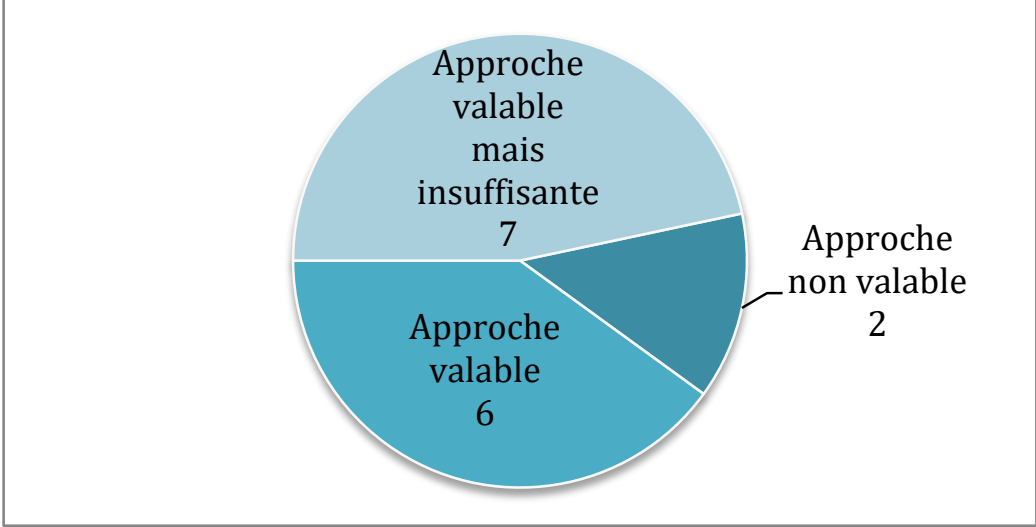

Seulement 6 enseignants trouvent cette approche valable. 7 enseignants sur 15 la trouvent valable mais insuffisante et deux enseignants trouvent cette approche non valable. Selon eux, d'abord le formateur ne peut pas tout programmer car l'homme loin d'être un automate apprend de plusieurs sources. Ce faisant, la formation peut induire des résultats positifs ou des résultats négatifs. L'issu n'est pas déterminé d'avance. Ensuite le second enseignant affirme qui dit, 'je n'enseigne rien à mes élèves, j'essaie seulement de créer des conditions dans lesquelles ils peuvent apprendre'” Pour ce qui est de l'application de cette théorie, la majorité des répondants reconnaissent appliquer cette méthode; comme le montre le graphique suivant : 


\section{Application : Thèse behavioriste}

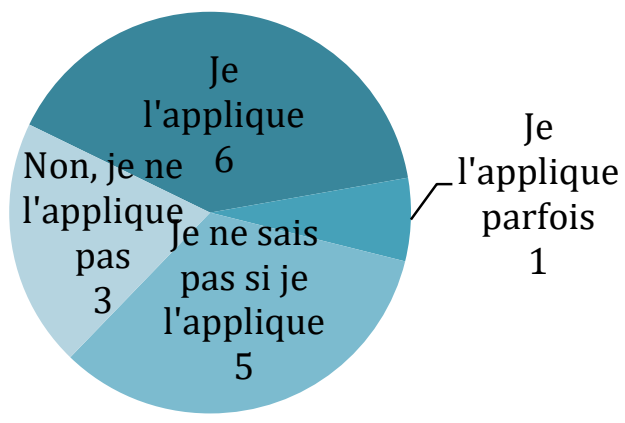

Parmi les 15 enseignants ayant répondu à nos questions, 6 affirment appliquer cette théorie dans leurs méthodes et un enseignant reconnaît l'appliquer occasionnellement. Par contre, 3 enseignants affirment clairement ne pas appliquer cette méthode dans leur approche d'enseignement. Par ailleurs, 5 enseignants ne se sont pas clairement prononcé sur l'application ou non de la thèse Behavioriste. Cette dernière catégorie de réponses s'expliquerait par les mêmes facteurs que dans le cas de la thèse constructiviste.

\section{3-3-Rapprochement aux théories sociocontructivistes de l'apprentissaqe}

\section{$>$ Thèse de Vyqotski}

Pour cette thèse, "Pour apprendre, il faut un médiateur, un facilitateur d'apprentissage et il faut aussi des obstacles organisés selon une graduation qui maintienne toujours la motivation de l'apprenant par l'accessibilité de ces obstacles. C'est l'envie de dépasser l'obstacle qui est le moteur de l'apprentissage. L'apprentissage tire sa source de l'interaction sociale importante du langage»

Selon les répondants, la thèse de Vygotski est une approche très valable dans la définition de l'apprentissage. Le graphique ci-après illustre bien ce fait. 


\section{Thèse de Vygotski}

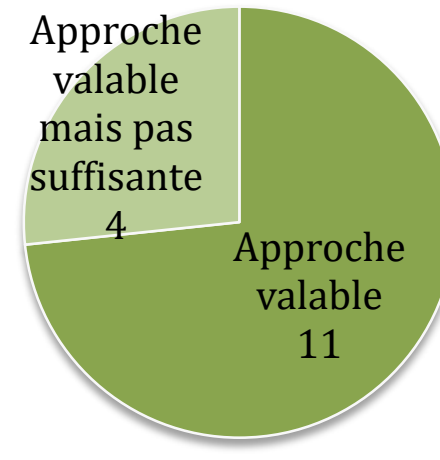

Pour 11 enseignants sur 15, Vygotski a une bonne approche de l'apprentissage. Certains de leurs contemporains (au nombre de 4), sans refouler la validité de l'approche de Vygotski estiment qu'elle n'est pas suffisante. En effet, c'est une théorie est applicable aux élèves ou étudiants d'un bon niveau qui cherchent à se perfectionner (Enseignant de Mathématiques). Ainsi, cette théorie ne correspondrait pas à l'élève moyen mais serait d'une grande utilité pour ceux qui se démarquent déjà du lot. Un autre enseignant, estime que le médiateur en question ne peut pas être qu'un enseignant. Il peut être un livre, des documents de références etc. (Enseignant de Sciences-Physiques). Du coup, pour parvenir à un apprentissage, nul besoin d'avoir un enseignant puisque par l'autodidaxie il est possible de parvenir à un apprentissage pertinent. Pour ce qui est de l'application, contrairement à la validité de la théorie, 6 enseignants affirment appliquer cette théorie tandis que 6 autres ne se pornoncent pas concrètement quant à l'application de cette théorie comme illustré sur le graphique suivant :

\section{Application : Thèse de Vygotski}

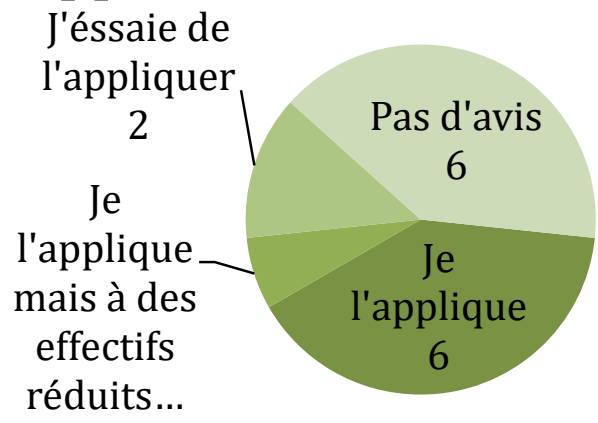


Par ailleurs, 3 enseignants reconnaissent qu'il n'est tout de même pas aisé de mettre en application cette théorie. Raison pour laquelle deux enseignants affirment déployer des efforts pour la mettre en œuvre au quotidien. Pour un autre enseignant, seuls les petits effectifs lui permettent de pratiquer une telle théorie.

\section{3-4-Rapprochement aux théories du traitement de l'information de l'apprentissage}

\section{Thèse du traitement de l'information}

Selon cette thèse, "On acquière des connaissances en traitant de l'information. Il accorde une place de choix à l'activité mentale et tente de construire des modèles de fonctionnement mental de l'apprenant, en utilisant des métaphores pour mieux décrire le processus en question»

Les avis sur la validité de cette thèse sont illustrés sur le graphique qui suit.

\section{Thèse du traitement de}

l'information Approche

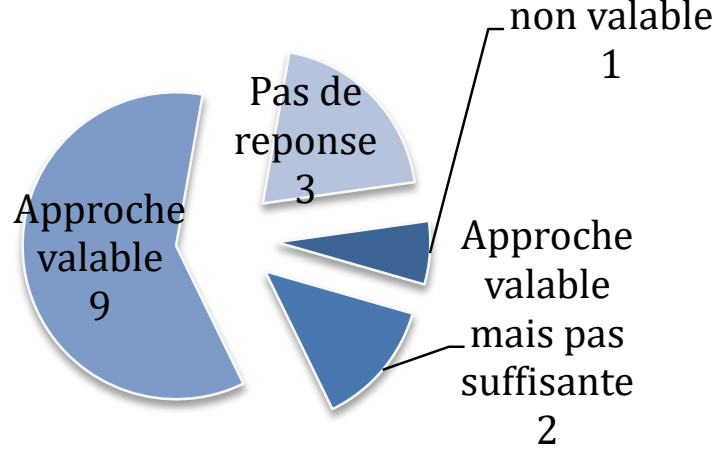

Parmi les 15 enseignants, 9 sont d'avis que l'approche du traitement de l'information est une approche valable de l'apprentissage. Notons ici que 3 enseignants n’ont pas répondu à cette question. Aussi, si pour deux enseignants cette approche n'est pas suffisante, un enseignant estime que le traitement d'information n'est pas une approche valable. Selon lui, l'activité mentale de l'apprenant n'est pas la résultante de la connaissance ou de l'information transmise. C'est dans la capacité à résoudre les exercices qu'on se rassure que l'activité mentale est bonne et parfaite. Ainsi, pour cet enseignant de Mathématiques, le traitement de l'information n'est pas le siège de l'apprentissage mais ce dernier réside dans l'exercice régulier. Autrement dit, le "'learning by doing'" est le meilleur moyen d'amener l'apprenant à un apprentissage pertinent. 


\section{3-5-Sont-ils logiques par rapport à ce que l'on attendait d'eux?}

La pertinence, la logique est-elle de mise, ou comment amener les apprenants à être compétents selon les problèmes posés par les enseignants ? Les études de cas vont supporter ces résultats.

La détermination d'une caractéristique de la Blatte se fait grâce à une démarche expérimentale. Une véritable démarche expérimentale dissocie les facteurs intervenant et ne fait varier qu'un facteur à la fois. La réalisation de l'expérience par l'apprenant passe par la détermination préalable du principe de l'expérience et par l'énoncé du dispositif expérimental

Le matériel d'étude est constitué de:

- $\quad$ bouteilles vides d'eau AWA

- thermomètres

- la glace fondante

- $\quad$ eau chaude

- 10 Blattes

- dispositif d’éclairement ampoule de 40 watts ou une torche.

AKO, 13 ans, $5^{\text {ième }}$.

Dans quels endroits as-tu récolté les blattes?

Sous les assiettes sales dans la cuisine, dans la douche et dans le W.C, sous le sommier du lit.

Dans quelles conditions sont les milieux où vivent les blattes?

Un milieu où elles peuvent se cacher de la lumière où elles peuvent trouver de la nourriture.

$Y$ - a-t-il d'autres conditions de milieu qui font que les blattes sont dans certains endroits?

A cause de l'humidité.

Si on te demande de montrer que les blattes sont sous le lit à cause de l'obscurité que vas-tu faire?

Je ne vais rien faire parce que qu'on est en classe.

Pourquoi ?

Parce que ce sera plus facile à la maison.

Il ne va rien faire parce qu'il ne se trouve pas à la maison. Les conditions de la salle de laboratoire sont impossibles à trouver puisque les éléments de la miniaturisation sont hors de sa portée. Il y a un défaut de la flexibilité mentale. La flexibilité mentale est définit selon Clément (2001) comme la capacité à changer de point de vue sur la situation (l'environnement) quand une première interprétation ne permet pas de trouver la solution (ne permet pas un comportement adapté). La plus ou moins grande facilité à changer de point de vue sur la situation explique les différences individuelles de performances dans la résolution de problèmes. 
Nous considérons ce changement de point de vue comme un indice crucial de la flexibilité mentale. lui.

Par contre, pour cet élève, il est en phase avec ce qui est attendu de

-ABDIAS, 14ans, $5^{\text {ième }}$.

L’élève parvient à faire la schématisation du dispositif expérimental et il arrive à trouver les différents éléments entrant dans sa composition. Elle parvient à dissocier les facteurs, donc à élaborer des hypothèses et des preuves. Chez cette dernière, la conception dès le départ du réel comme un produit de divers facteurs se composent d'un ensemble de combinaisons possibles.

Enseignant : Tu vas mettre le cafard à quel endroit?

Elève: Comme on dit qu'il ne peut pas supporter la lumière je vais le mettre d'abord du côté de la lumière

Dans la lumière, il va faire quoi ?

Il va essayer de fuir, de s'échapper maintenant; pour entrer tout ça, je vais les mettre dans le coin sombre avant de les y fermer.

L’observation scientifique est très élaborée par l'esprit parce que le fait de la connaissance courante, celui de la simple perception est construit, en ce sens qu'il n'y a pas de "fait brut". Le fait scientifique est plus élaboré et il va résulter d'un choix et partant certains parlent de "faits privilégiés", de fait interprété voire de fait rectifié. Ensuite, elle va confronter les faits à l'expérience à la réalité scientifique avérée pour savoir s’il est vérifiable.

Comment vas-tu éclairer le milieu?

Je vais chercher une ampoule de 60watt ou 100watt

Une ampoule de 60 watt ou 100 watt, oui !

Je vais chercher un truc comme çà (Il fait une image qu'il décrit dans l'espace comme étant une canne avec l'extrémité terminée par une ampoule) et mettre le carton, c'est-à-dire, l'ampoule est fixée directement sur le carton-là. Maintenant, je vais brancher la partie sombre et la partie éclairée...

...Eclairée oui ! Ensuite tu vas faire quoi ?

D’abord, je vais mettre le cafard dans la partie éclairée puisque j’ai déjà fait la limite où il y a la partie sombre et la partie éclairée. Donc, je vais essayer de faire entrer le cafard par une petite porte que je dépose. Ensuite, vous allez voir quand la lumière va frapper il va chercher un coin, où il va s'échapper. C'est pour ça que quand il va regarder, qu'il va voir une petite porte où le coin est sombre, il va chercher à aller là-bas.

Maintenant, tu veux faire une expérience pour montrer si les cafards aiment les endroits où il y a l'eau, les endroits mouillés, cela veut dire quoi ?

Ils aiment l'eau 
Ils aiment l'eau, qu'est-ce qu'ils n'aiment pas?

Ils n'aiment pas les endroits secs.

Ils n'aiment pas les endroits très secs, ils aiment les endroits mouillés, où il y a de l'eau. Si toi tu veux étudier, si toi tu nous montres que les cafards aiment les endroits humides et qu'ils n'aiment pas les endroits très secs, comment tu vas faire ton expérience?

Je vais utiliser sous forme de carton un bocal en verre

En quoi ?

\section{Un bocal en verre}

Un bocal en verre, tu veux dire transparent?

Oui ! Transparent, je vais essayer de mettre un côté, pas d'eau dedans, je vais essayer d'y mettre de l'eau et un côté très sec. Je vais y mettre du sable très sec, il n'y a pas de l'eau là-bas. Je vais essayer de diviser la partie comme j'ai eu à le faire pour le carton au cours de l'autre expérience. On va mettre le cafard dans le milieu très sec et le cafard ne va pas être à l'aise, il va se dire ah! Je cherche un milieu humide alors que le milieu est très sec, il va chercher à trouver un endroit très humide. C'est cet endroit où il va entrer parce que il va voir que ah! L'endroit qui est là est humide!

Les sujets parviennent à dissocier les facteurs, donc à élaborer des hypothèses et des preuves. Les sujets arrivent à dissocier les facteurs, mais surtout ils arrivent à établir les correspondances données dans l'expérience immédiate. Ils se reconnaissent à l'apparition d'un raisonnement hypothécodéductif en même temps qu’à une recherche active de vérification. La prise de conscience permet de regarder les choses autrement et ainsi le pouvoir d'agir différemment sur elles, "comme devant le jeu Awalé : je vois autrement, je joue autrement". Cependant on peut reconnaître que le simple fait d'être conscient de quelque chose dont on n'était pas conscient auparavant change la perception du monde et agit peut-être, sans qu'on en soit conscient, sur sa manière de faire. Autrement dit pour Vygotski (1997), je peux ne pas vouloir être attentive à ma perception auditive ou à mon mode d'évocation. A partir du moment où je suis conscient que ces gestes mentaux existent, ils peuvent "s'imposer à mon attention" malgré moi : nommer les choses, c'est peut-être déjà les faire exister de façon autonome.

Donc c'est là-bas il va partir!

Oui, c'est là-bas il va partir ! Donc, ma conclusion est que les cafards ne peuvent rester dans un milieu très sec, il aime les coins humides.

Maintenant, tu veux faire une expérience pour montrer si les cafards aiment la chaleur. Si les cafards aiment la chaleur cela veut dire quoi ?

Ils n'aiment pas la fraîcheur 
Ils n'aiment pas la fraîcheur, ils n'aiment pas le froid, alors si on te dit de montrer par une expérience que les cafards aiment les endroits chauds où il y a la chaleur, de ceux où il y a le froid, que vas-tu faire?

Je vais prendre un cas de notre douche pour bien faire l'expérience le jour où j'ai envoyé les cafards. Je vais faire l'expérience quoi ! Parce que un jour j'ai fait une expérience laissée libre que ah! Le cafard n'est pas dedans! Je vais bien expliquer! Dans le cas de notre douche on a une petite porte ici et tout çà la nuit on ferme. Le jour où vous avez dit d'apporter les cafards la journée j'ai cherché les cafards la journée ils n'étaient pas là ! Mais dès que nous avons fermé, parce que nous avons un climatiseur la chaleur donne sur la douche, il frappe directement sur la douche et il y a la chaleur, c'est la nuit que je me suis assis...

Quand met-on en marche le climatiseur?

C'est la nuit

C'est la nuit qu'il dégage la chaleur

Oui ! Notre climatiseur est déposé de sorte qu'il dégage de la chaleur. Je me suis arrêté je me suis dit ah! Mon professeur a dit d'apporter des cafards et depuis-là je cherche les cafards et on ne les trouve pas. Donc on n'a qu'à essayer la nuit pour voir c'est pour ça j'ai attendu, j'ai vu que ah beaucoup de cafards à rentrer dans la douche que le climatiseur dégage de la chaleur et j'ai vu eux tous sont entrés dedans. Je me suis dit ah ! Mon petit frère comme ils sont entrés allons-y prendre pour les mettre dans la bouteille; c'est dans ça, j'ai vu que les cafards n'aiment pas la fraîcheur mais ils aiment la chaleur.

Les cafards aiment la chaleur, maintenant au laboratoire, ici tu vas nous montrer que les cafards n'aiment pas la fraîcheur et ils aiment la chaleur !

Je vais prendre un petit endroit, un milieu comme ça, est très chaud ! Je vais prendre un bocal qui va durer pendant très longtemps comme il n'y a de l'air pour que l'air chaud se dégage. L'air chaud est dans le bocal pendant longtemps, il n'y a pas de fenêtre, il n'y a rien.

Tu as fermé tout.

Oui ! J'ai fermé tout. J'ai creusé un petit trou pour que ça passe directement dans cet autre bocal donc l'air ne peut pas détacher, il est enfermé. Si par exemple je prends un cafard je le mets là où il y a la fraîcheur et je précise, là où il y a le bocal où il y a la chaleur. Je vais percer le côté...

Tu as ce vase, tu vas faire un côté où il y a la chaleur et de l'autre côté tu as de la fraîcheur, là où il y a de la fraîcheur, comment tu vas t'arranger pour qu'il y ait de la fraîcheur, là seulement où tu veux qu'il y a la fraîcheur ?

Si ici est fermé je vais essayer de faire une limite (Il met une limite passant par le centre) comme la première expérience et de ce côté-ci (A gauche de la... ) l'air peut aller de bas en haut... 
L'air peut aller de bas en haut, mais est-ce que tu peux utiliser pour mettre vraiment de la fraîcheur pour qu'il fasse froid?

Je vais chercher une glace

La glace bien!

Je mets la glace en dessous de la partie droite et ce sera bien glacé. C'est du caoutchouc, en bas sera glacé, on va prendre un cafard on va le mettre dedans il va chercher un côté où il y a de la chaleur. Alors que, où j’ai limité l'air ne peut pas sortir parce que l'air est très chaud. Par le trou que j’ai creusé il va chercher à se mettre du côté de l'air chaud. Donc je peux dire que les cafards aiment les endroits où il y a de la chaleur.

Ces derniers conçoivent, dès le départ, le réel comme un produit de divers facteurs. Ces divers facteurs vont avoir, entre eux, une sorte d'ensemble de combinaisons possibles. Dès lors, qu'il y a apparition de l'hypothèse, cela va consister à reconstituer ces combinaisons et à établir effectivement les liens de conformités d'expérience.

Chez eux, il y a existence d'un plan expérimental qui a requis l'usage de la pensée formelle. Ces derniers ont pu émettre des hypothèses, prévoir les résultats et les vérifier expérimentalement. Les difficultés au niveau de la conceptualisation du dispositif expérimental sont résolues assez aisément par le sujet. La conception du protocole expérimental ne pose pas de problème d'imagination et de créativité. Car elle emprunte des opérations intellectuelles qui rendent compte du fait que la démarche apparaît clairement à l'apprenant et en plus celui-ci ne prend pas conscience des démarches intellectuelles qu’il doit mobiliser pour réaliser la tâche.

L'entretien entre l'apprenant et l'enseignant se déroule sur un plan inter-psychologique. L'enseignant pose des questions et l'apprenant essaie d’y répondre en conformité avec son savoir et ses connaissances. Au cours de ces séances l'apprenant essaie d'avoir tous les rudiments qu'il faut, afin de parvenir au niveau intellectuel que lui permettent les interactions enseignant-enseigné.

\section{4-Discussion et conclusion}

Cet article est une contribution à la compréhension du fonctionnement du système éducatif ivoirien et en particulier du processus d'enseignement de même que du processus d'apprentissage. D'entrée de jeu, nous sommes posés quelques questions fondamentales : Les apprenants sont-ils devenus pertinents ? Sont-ils logiques par rapport à ce que l'on attendait d'eux ? Quel type d'évaluation peut-on en faire des enseignements dispensés en rapport aux théories d'apprentissage?

L'hypothèse formulé au départ suppose la pertinence de l'apprentissage des apprenants du système ivoirien. Loin de se vouloir inscrit dans un cadre plus général, l'étude que nous avons réalisée s'intéresse 
singulièrement au cas de l'établissement Jean Piaget. Au total, 15 enseignants et 26 apprenants ont répondu à notre questionnaire.

Notons que dans l'ensemble, les apprenants reconnaissent être plus ou moins informé des attentes des enseignants en lors de leurs premiers contacts. De ce fait, les apprenants, dans une modélisation idéaliste devraient se disposer à répondre au mieux aux attentes de leurs enseignants. Selon les divers avis recueillis, plus de la moitié des apprenants n'appliquent pas à la lettre ou n'appliquent pas toujours les conseils et autres astuces des enseignants. De cet angle de vue, il existe un manque de logique au niveau des apprenants par rapport à ce qui est attendu d'eux.

Le pont que nous avons tenté d'établir entre l'enseignement dans le système éducatif ivoirien et les théories d'apprentissage. Il apparaît que la thèse constructiviste est celle qui de loin est plus perceptible par les enseignants dans leur manière d'enseigner. Toutefois, toutes les thèses présentées dans ce papier sont jugées valables par la plupart des enseignants. Ainsi, le premier obstacle entre l'enseignement dispensé dans notre système et la théorie est la conscience au niveau des enseignants eux-mêmes. En effet, l'on constate que les enseignants ne perçoivent pas suffisamment le fondement théorique de la construction de leur enseignement. De plus, on note une instruction insuffisante sur les théories que nous leur avons présentées.

Il y a bien sûr d'autres modèles que les 4 présentés ci-dessus et il faut aussi considérer les caractéristiques individuelles des apprenants. Souvent en pratique, les enseignants naviguent d'un modèle à l'autre. Il faut savoir choisir un modèle en fonction d'un certain nombre de paramètres. Finalement, il s'agit d'être conscient de la conception sur laquelle on a naturellement tendance à s'appuyer, ainsi que de l'existence d'autres conceptions de façon à pouvoir diversifier son enseignement.

Toute pratique enseignante repose sur des présupposés psychologiques car, elle concrétise, pour une part, les conceptions de l'apprentissage implicites ou explicites de l'enseignant. Si ce dernier choisit de dire et de montrer le savoir, c'est qu'il présume, consciemment ou inconsciemment, qu'ainsi il favorisera l'apprentissage du plus grand nombre possible de ses élèves dans le temps imparti et qu'il permettra les apprentissages ultérieurs. S'il préfère une fiche d'activités où des questions relativement faciles s’enchaînent jusqu’à la "découverte" du savoir visé, c’est qu'il prétend que dire le savoir ne suffit pas. C’est aussi qu’il pense que sa fiche d'activités, ainsi conçue, facilitera davantage la construction du savoir de ses élèves ou aidera à l'apprentissage d'un plus grand nombre d'élèves, ou encore, fera moins obstacle aux futurs apprentissages. S'il suit une autre voie encore, c'est qu'il suppose que les deux premières ne conviennent pas et qu'il faut donc envisager un autre mode d'enseignement. 
Dans l'idéal transmissif, l'enseignant mais aussi l'élève ne doivent pas se tromper. En effet, l'erreur pourrait créer de mauvais réflexes ou s'imprimer dans la tête de ce dernier. Il faut donc dresser un barrage à l'erreur. Si toutefois un élève commettait une erreur, la seule remédiation possible serait d'expliquer à nouveau ou de refaire apprendre en lui demandant d'être plus attentif. Et, en dernier ressort, de faire redoubler l'élève pour de nouvelles explications.

Dans toutes les conceptions inspirées du béhaviorisme, l'enseignement est fondé sur le découpage des connaissances. L'élève progresse pas à pas, l'apprentissage étant renforcé par des constats de réussite. L'erreur est donc à éviter. Si, toutefois elle survenait, c'est que l'élève n'aurait pas maîtrisé certains prérequis indispensables. Ou que la connaissance n'aurait pas été décomposée en éléments suffisamment petits pour être confondue avec une réponse adaptée à un stimulus.

Pour Piaget, l'apprentissage reste une relation privée entre un sujet, les objets, la tâche, le problème. Les relations sociales entre pairs ou avec un éducateur ne semblent pas prééminentes dans le développement cognitif. Dans ces conditions, on voit mal la place de l'enseignement dans ce développement. Dans les conceptions constructivistes de l'apprentissage, l'erreur est le résultat de processus d'origine sensée. Il n'est plus question d'y faire barrage, mais, au contraire, elle apparaît comme normale à l'apprentissage. Elle est l'expression ou la manifestation explicite d'un ensemble de conceptions intégrées dans un réseau cohérent de représentations cognitives, qui se dressent en obstacles à l'acquisition et à la maîtrise de nouveaux concepts. Le franchissement de ces obstacles devient alors le projet de l'acte d'enseignement et l'erreur un épisode dans la restructuration et l'élargissement des connaissances. " La compréhension s'acquiert contre une connaissance antérieure en détruisant des connaissances mal faites ». Les approches à privilégier dans toutes les matières au programme sont celles qui donnent un sens aux apprentissages de par la pertinence des contenus proposés. Les approches retenues doivent permettre l'interaction et la collaboration entre les élèves, expérience décisive dans la construction des savoirs. Dans ce contexte l'élève travaille dans une atmosphère de socialisation où les talents de chacun sont reconnus. L'enseignement doit favoriser l'interdisciplinarité en vue de maintenir l'habitude chez l'élève de procéder aux transferts des savoirs, des savoirfaire et des savoir- être. L'apprentissage doit doter l'élève de confiance en ses habiletés afin qu'il s'investisse pleinement dans une démarche personnelle qui lui permettra d'atteindre un haut niveau de compétence.

Les théories d'apprentissage entre autre, permettent d'opérationnaliser l'analyse des processus cognitifs complexes en rapport avec les connaissances et la mémoire. L'analyse des concepts de 
connaissances et de résolution de problème, au travers de la psychologie cognitive fournit une base importante à la compréhension même du concept de problème. Il y aurait selon Clément (2001) des différences de flexibilité mentale dans l'activité de résolution de problèmes que n’abordent pas les théories d'apprentissage. Ainsi, "la résolution de problème est conçue comme une suite de restructurations concernant essentiellement le champ perceptif". Toute activité cognitive peut être comprise comme une activité de résolution de problème si l'on accepte que la cognition soit intentionnelle, dirigée par l'atteinte d'un but et l'évitement des obstacles à ce but (Anderson, 1995). Mais, il y a le rôle fondamentale joué la représentation de l’apprenant. La représentation du sujet-apprenant est "formalisée par une liste de contraintes hiérarchisées qui autorise certaines actions et en interdit d'autres".

Est-ce un leurre psychologique ou revêt-il un caractère pertinent, un caractère logique du système éducatif face aux théories d’apprentissage?

Cette pertinence et cette actualité revisitées peuvent être affirmées dans le domaine de la réforme en pédagogique en général où des processus cognitifs seront développés par des sujets.

\section{References:}

Anderson, J. R., (1995). Cognitive Psychology and its Implications. New York : Freeman and Co.

Bruner, J., (2000). Cultures et modes de pensée. L'esprit humain dans ses œuvres. Paris : Retz.

Clément, E. (2001). Etude des différences de flexibilité mentale dans l'activité de résolution de problèmes. In A. Flieller, C. Bocéréan, J-L Kop, E. Thébaut, A-M. Toniolo, J. Tournois, Questions de psychologie différentielle, (pp 317-322). Rennes : Presses Universitaires de Rennes.

Clot, Y.,(2002). Avec Vygotski. Paris,La Dispute/SNEDIT.

Jonnaert, Ph., (2002). Compétences et socioconstructivisme. Un cadre théorique. Bruxelles : De Boeck.

Jonnaert, P., et Vander Borght, C., (2003). Créer des conditions d'apprentissage. Un cadre de référence socioconstructiviste pour une formation didactique des enseignants. Bruxelles, de Boeck Université.

Montmollin M. de, 1986. L'intelligence de la tâche. Eléments d'ergonomie cognitive. Berne: Peter Lang.

Piaget, J. et coll., (1967). Logique et connaissances scientifique. Paris. Gallimard: collection La Pléiade.

Schneuwly, B., et Bronckart, J.P.,(1985). VYGOTSKY aujourd'hui. Delachaux et Niestlé

Vygotski, L.,(1997). Pensée et Langage, éditions Paris, La Dispute. 